\title{
Methods for Simulation of Electron Energy Loss Spectra for Low Energy Edges in Battery Materials
}

\author{
Quentin Stoyel $^{1}$, Frederic Voisard ${ }^{1}$, Nicolas Brodusch ${ }^{1}$, George Demopoulos $^{1}$ and Raynald Gauvin ${ }^{1}$ \\ 1. Mining and Material Engineering, McGill University, Montreal, Canada.
}

Lithium ion batteries are essential to many components of society from supporting the smart phone revolution to the development of batteries as solutions for minimizing climate change. In order to continue to further improve batteries, characterization techniques of these materials must become more refined. Electron microscopy is a tool that can fill this role, offering a wealth of improving capabilities as well as a number of versatile characterization methods. Amongst these, electron energy loss spectroscopy (EELS) presents great potential to take advantage of recent advances and become a key method in the characterization of battery materials. Improving energy resolution and the large variety of data that can be obtained (chemical environment, compositional, bond strength, etc) make EELS an attractive choice for characterization.

One of the challenges however, lies in the nature of battery materials being sensitive to both beam damage and charging. Improving microscopes allow us to minimize these issues to some extent, such as McGill's SU 9000 capable of acquiring EELS at $30 \mathrm{keV}$, allowing for the detection of lithium. This technological improvement can be further complemented through strong theoretical support. In the case of EELS, cutting edge experimental results can be compared to density functional theory (DFT) calculations for solid state analysis. By calculating either the cross section or the energy loss function it is possible to obtain first principle simulations of the spectra [1]. As DFT calculations are unaffected by experimental constraints such as beam damage or synthesis processes, they are an attractive and efficient tool both to support analysis and to assist in predicting improved materials.

Theoretical simulations of the lithium K edge ( $\sim 55 \mathrm{ev})$ or the M 23 edges of transition metal needed to characterize battery materials, present a range of challenges to DFT simulation [2]. The low energy nature of these edges push the limits on some of the assumptions used to calculate EELS spectra. Many of these approximations focus on how to deal with excitonic effects, amongst others, the core hole approximation. This approximation involves manually moving a core electron to the conduction band in order to simulate an excited state. In some cases, this can dramatically improve agreement with experiment, such as in LiF (Fig 1). However the core hole approximation is sometimes insufficient, unnecessary, or excessive as can be seen in the inability to reproduce the correct peak ratios when applied to $\mathrm{Li}_{2} \mathrm{O}$ (Fig 2) and while some corrections have been suggested, there is not yet a consistent means of applying the approximation [2,3]. The low energy Li K edge also introduces more challenges due to it's loosely bonded electrons. Lithium's disperse electron cloud make it vulnerable to monopole effects, which must also be taken into account in simulating the spectra [4]. Finally, the importance of accurately simulating the lithium K edge is further necessary when considering its overlap with the M23 edges of the transition metals present in battery materials, such as Fe, Ni, Co, and Mn [2]. Whilst some of these challenges can be solved through the use of a two particle simulation (solving the Bethe-Salpeter equations) it is a computationally heavy task which make it impractical for the timely support of experiment [3]. In order become a meaningful tool for the analysis of new experimental results, a consistent means for handling the multiple challenges associated with low energy edges is needed. These assorted challenges are discussed and evaluated in the 
context of simulating low energy edges in battery materials, with the goal of developing a robust and rapid method to support experiments [5].

References:

[1] C. Hebert, Micron 38(1) (2007), p. 12.

[2] V. Mauchamp et al, Phys. Rev. B 74 (11) (2006), p. 115106.

[3] T. Mizoguchi et al, Ultramicroscopy 180 (2017), p. 93.

[4] V. Mauchamp et al, Phys. Rev. B 77(4) (2008), p. 045117.

[5] The authors acknowledge funding from McGill University, NSERC CGS, and IREQ.

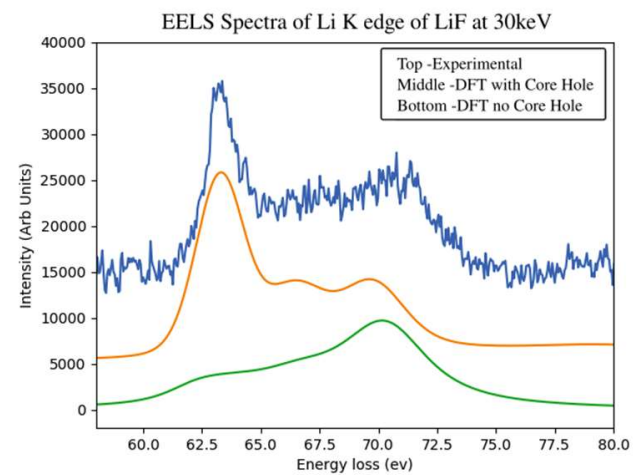

Figure 1. Calculated and experimental spectra of Li K edge of LiF. Calculation performed with WIEN2k TELNES3 module, core hole inserted in a 2x2x2 supercell. Experimental Spectra from McGill University taken at $30 \mathrm{keV}$.

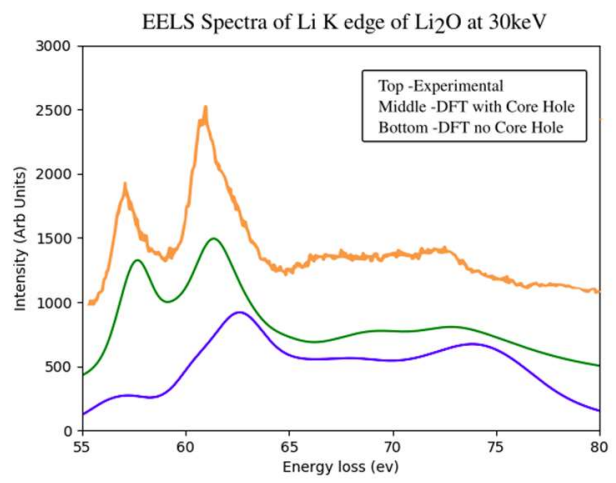

Figure 2. Calculated and experimental spectra of $\mathrm{Li} \mathrm{K}$ edge of $\mathrm{Li}_{2} \mathrm{O}$. Calculation performed with WIEN2k TELNES3 module, core hole inserted in a 2x2x2 supercell. Experimental Spectra from McGill University, taken at $30 \mathrm{keV}$. 